\title{
Experimental Study of High-speed Rough-terrain Mobile Robot Models for Reactive Behaviors
}

\author{
Karl Iagnemma, Dariusz Golda, Matthew Spenko, and Steven Dubowsky \\ Department of Mechanical Engineering, Massachusetts Institute of Technology \\ Cambridge, MA 02139 U.S.A.
}

\begin{abstract}
High-speed mobile robots have many important applications in rough terrain. At high speeds, it is difficult to guarantee safe robot motion using traditional control and planning techniques. This paper presents an experimental high-speed rover system for studying reactive behavior control to avoid potentially dangerous situations. The method consists of sensor-triggered maneuvers that have been shown by a priori model-based analysis to be safe. The paper discusses vehicle and terrain models for model-based analysis. Experimental results show that behaviors based on detailed models can accurately predict the dynamics of mobile robots in rough terrain.
\end{abstract}

\section{Introduction}

Mobile robots are being developed for applications in rough terrain, such as planetary exploration, mining, and hazardous site inspection [2, 5, 6, 10] (see Figure 1). In many applications, including military reconnaissance, it would be desirable that the robot move at high speed [4]. At high speed, sensor uncertainty and limited terrain data can result in unexpected and dangerous situations.

In this work a low-level "reactive behavior" control method that can effectively respond to unexpected situations is studied. The method would detect and avoid hazards that are not anticipated by higher-level control and planning layers. In this architecture, a global planning algorithm would plan a route through a largescale (i.e. km-level) terrain, based on gross terrain elevation knowledge. A local planning algorithm would then refine the motion plan based on vision sensor information, including range data, while the robot is traveling. The reactive behavior control would compensate for unexpected or unmodeled robot motions, based on proprioceptive sensor feedback. 


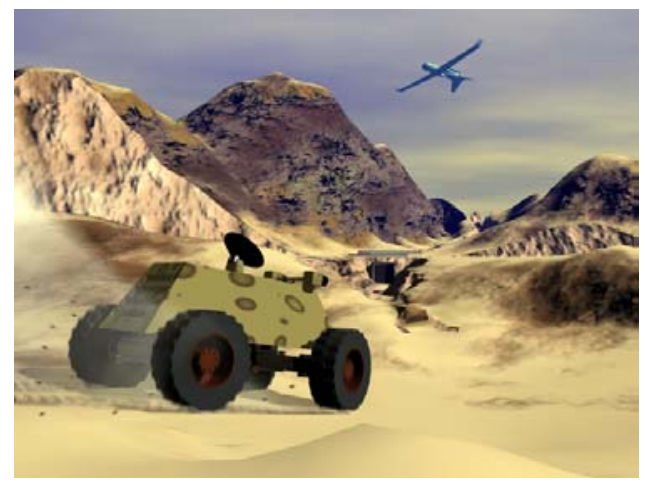

Fig. 1. High-speed mobile robot in rough terrain

A reactive behavior is a sensor-triggered maneuver, chosen from a small library of pre-defined maneuvers, such as emergency stops, hard turns, braking turns, etc., to produce a safe response to unexpected conditions. These behaviors are developed a priori using analysis of vehicle and terrain models. The experimental results presented in this paper suggest that detailed vehicle and terrain models are required to accurately predict high-speed robot performance. However, detailed on-line model-based analysis is not computationally feasible at high-speed. The proposed method therefore uses a library of predicted dynamic behaviors built from off-line analysis of rover performance under various vehicle and terrain conditions.

Few researchers have addressed the problem of model-based analysis of highspeed robots in rough terrain. Substantial research has been performed in the control of high-speed vehicles on nearly flat terrain [11]. However, simplifications made are not valid in rough terrain. Research has also shown that vehicle dynamics and controller selection can have substantial influence on robot behavior [1]. Other researchers have studied predictive control of a mobile robot in uneven terrain at moderate speeds [9]. This work, however, assumed that the vehicle did not experience wheel slip or lose contact with the terrain, which is generally not true at high speeds. In summary, few researchers have studied model-based analysis, including terrain effects, for high-speed rough-terrain robot control.

In this paper, the concept of reactive behavior control is briefly presented, along with its underlying model-based analysis for a four-wheeled vehicle. The reactive behavior control is similar to the idea of maneuver automatons introduced by Frazzoli for autonomous aerial vehicles [3]. A description of the high-speed rover experimental testbed used to study this apporach is presented. Vehicle and terrain models of varying complexity are described. Simulation and experimental results are compared for a four-wheeled vehicle crossing a ditch at a high-speed. This study suggests that the motion dynamics of high-speed mobile robots in rough terrain can be accurately captured by off-line vehicle and terrain models. 


\section{Reactive Behaviors for High-Speed Mobile Robots}

Here a reactive behavior control scheme for a four-wheeled mobile robot with one drive input (i.e. throttle), $\in \mathrm{m} \mathrm{m}$, and one steering input, $\in \mathrm{m}_{\mathrm{m}}$ is presented (see Figure 2). For this system, a number of reactive behaviors can be defined, for example: constant-speed turn $\left(={ }_{c} ; \quad \pm_{m}\right)$; braking turn $(=-\mathrm{m}$;

$\pm_{m}$ ); emergency stop $(=\mathrm{m} ; \quad c)$. The subscript $c$ represents the current actuator value. Other, more complex reactive behavior classes can also be defined, including: yaw-rate stabilization, to avoid excessive sideslip; ballistic stabilization, to avoid excessive pitch when contact with the terrain is lost; etc.

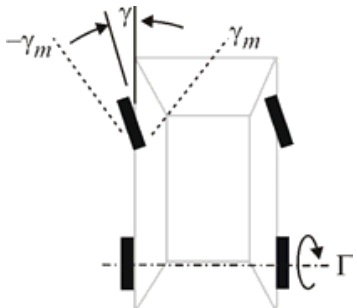

Fig. 2. Diagram of four-wheeled mobile robot control inputs

Reactive behaviors are triggered by on-board sensors. It is assumed that the robot can measure the following: vehicle roll, pitch, and their rates; wheel angular velocities; range to terrain features within several vehicle lengths; estimates of the terrain roughness (from vision sensors and elevation data) and soil properties such as cohesion and internal friction angle computed from on-board sensors [7, 8].

When a potentially hazardous situation is detected, a reactive behavior that would avoid the hazard is selected. For example, if a negative obstacle was detected at close range, the reactive behavior "Constant Speed Turn" might be selected (see Figure 3).

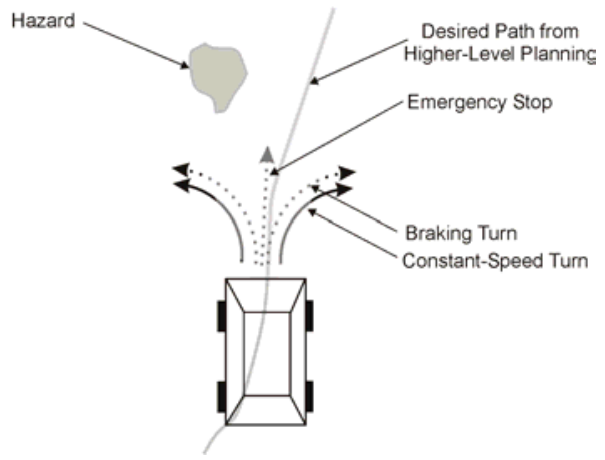

Fig. 3. Example of reactive behavior selection 
These maneuvers are strongly dependent on vehicle dynamic properties, vehicle pose, and terrain properties. The focus of this work was to determine experimentally the model complexity required to yield valid high-speed behavior prediction. The rover testbed used in this experimental study is described below.

\section{Experimental System Design}

The experimental testbed consists of an on-board sensor suite, computer, electronics, and communications hardware mounted on a high-speed rough-terrain vehicle (see Figure 4). Differential GPS and an inertial measurement system provide vehicle position and state information. A stereo camera pair provides terrain range, color, and texture information. Wheel tachometers and suspension potentiometers provide wheel state information. A fixed-base computer controls experiments via a user interface. A radio modem provides wireless communication.

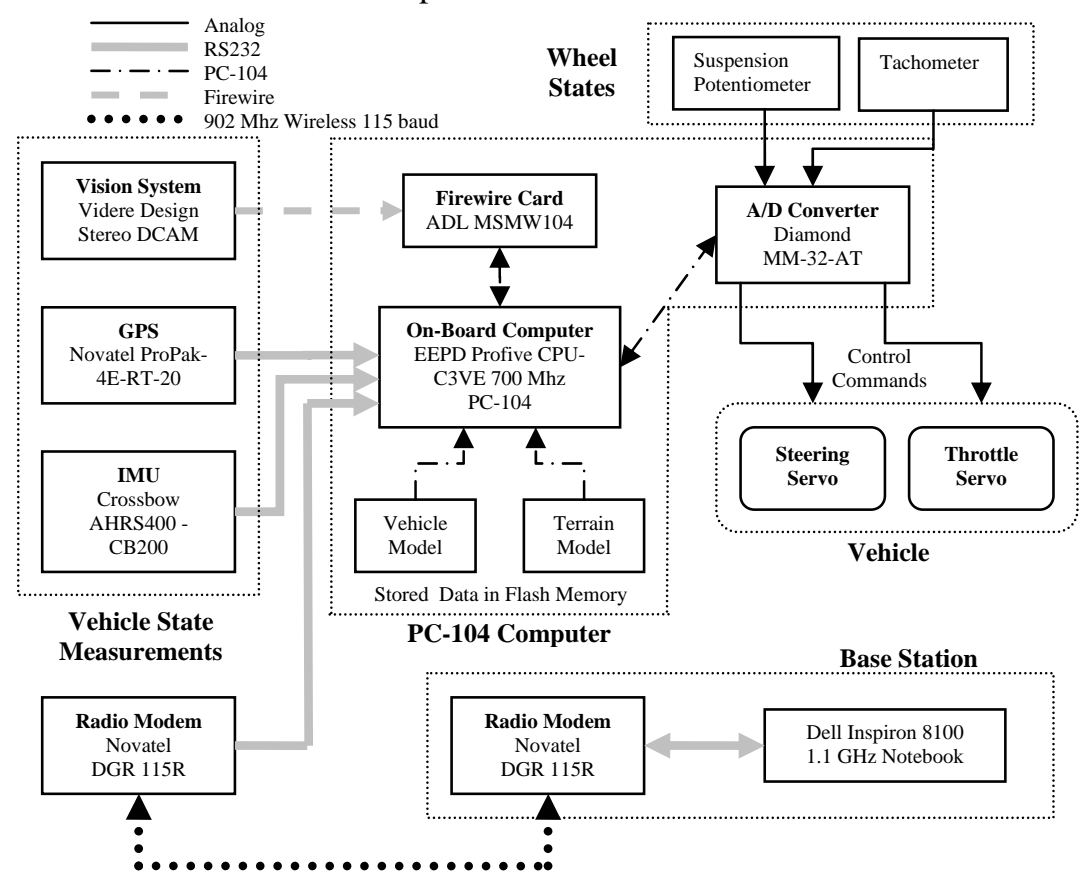

Fig. 4. Experimental testbed schematic 


\subsection{MOD 1 High Speed Vehicle}

A 1/10 scale electric powered vehicle was selected for model validation experiments (see Figure 5 and Table 1). It is tele-operated, with two control inputs: a rear-wheel DC motor drive input, and a front wheel steering servo. The rear wheels are coupled by a differential, and the front wheels are joined by an Ackerman steering linkage. The rover has tunable independent, spring-damper suspensions for the wheels, and deformable rubber tires. For model validation, this rover does not need to carry the full sensor and computation package described above. A three-axis accelerometer and flash memory module are mounted to the vehicle for recording body accelerations.

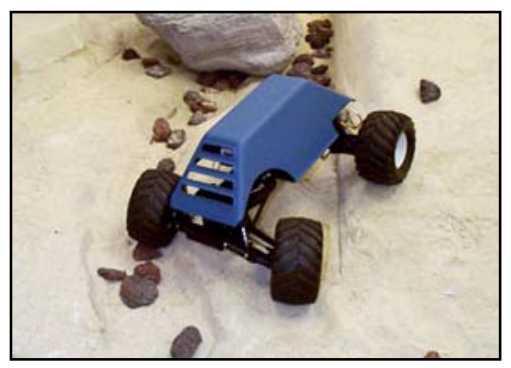

Fig. 5. MOD 1 experimental rover

Table 1. MOD 1 experimental rover physical parameters

\begin{tabular}{ll}
\hline Mass & $3.1 \mathrm{~kg}$ \\
Dimensions (Length x Width) & $0.30 \mathrm{~m} \mathrm{x} 0.30 \mathrm{~m}$ \\
Longitudinal Axis Moment of Inertia & $0.0065 \mathrm{~kg} \cdot \mathrm{m}^{2}$ \\
Transverse Axis Moment of Inertia & $0.022 \mathrm{~kg} \cdot \mathrm{m}^{2}$ \\
Yaw Axis Moment of Inertia & $0.023 \mathrm{~kg} \cdot \mathrm{m}^{2}$ \\
Wheel Radius, Width & $0.061 \mathrm{~m}, 0.066 \mathrm{~m}$ \\
Wheel Stiffness, Damping & $6000.0 \mathrm{~N} / \mathrm{m}, 18 \mathrm{~N}-\mathrm{s} / \mathrm{m}$ \\
Suspension Stiffness, Damping & $500 \mathrm{~N} / \mathrm{m}, 74.1 \cdot v^{2}+41 \cdot v \mathrm{~N}-\mathrm{s} / \mathrm{m}$ \\
& $(v$ is suspension compression rate $)$ \\
\hline
\end{tabular}

A high-speed gasoline-powered rover is currently being developed, and will carry the full sensor and computation package described above (see Figure 6 and Table 2). It has a top speed of $27 \mathrm{~m} / \mathrm{s}$, and will be controlled via two inputs: throttle to the engine driving the rear wheels, and a front wheel steering servo. The rear wheels are mounted to single drive axle, and the front wheels are joined by an Ackerman steering linkage. The front suspension is an I-beam configuration, while the rear suspension is a four-link trailing arm design. Both front and rear suspensions are tunable spring-dampers. The vehicle has rubber tires. Braking is achieved with a clutch-mounted friction brake. 


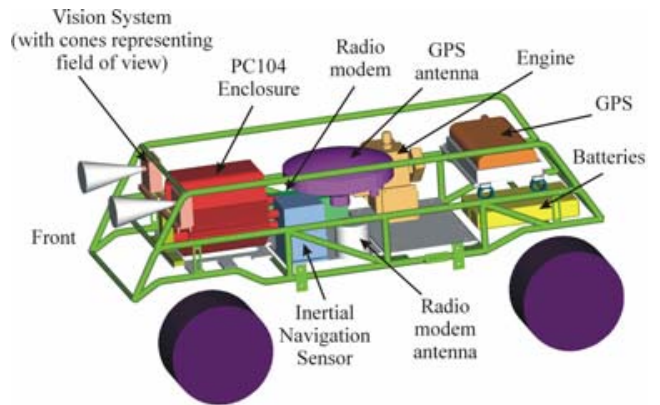

Fig. 6. MOD 2 experimental rover

Table 2: MOD 2 rover properties

\begin{tabular}{ll}
\hline Mass & $23 \mathrm{~kg}$ \\
Dimensions (Length x Width x Height) & $(0.89 \times 0.31 \times 0.23) \mathrm{m}$ \\
Wheel Radius & $0.19 \mathrm{~m}$ \\
Top Speed & $27 \mathrm{~m} / \mathrm{s}$
\end{tabular}

\section{Robot and Terrain Models}

To study high-speed reactive behaviors, two dynamic analysis software packages, RAD and ADAMS were used. RAD is dynamic analysis software that was developed for this study, and uses a dynamic solver based on a Newton-Euler formulation, with a fourth-order Runge-Kutta integration method. ADAMS is commercial dynamic analysis software, which allows simulation of CAD-specified vehicle models, and sophisticated wheel-terrain interaction models. Both vehicle models contained fifteen degrees of freedom: three translational and three rotational DOF for the body, one translational and one rotational DOF for each of the wheels, and one steering angle (see Figure 7). The model considers the mass and inertia of the body, wheels, and suspension, and independent spring-damper suspensions.
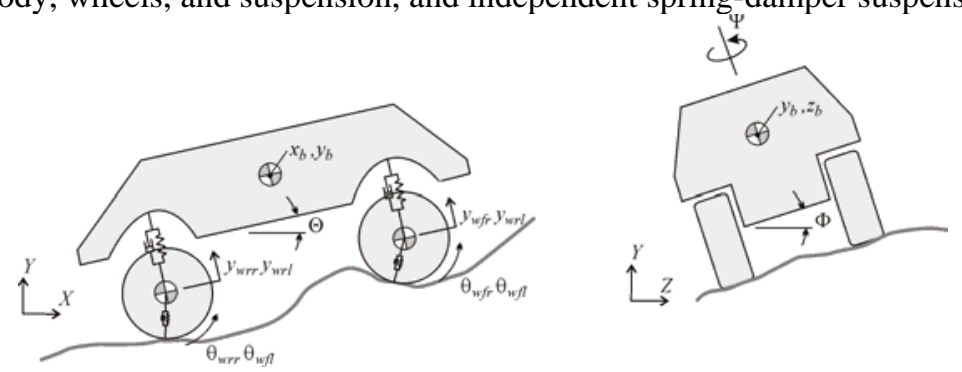

Fig. 7. Diagram of fifteen DOF vehicle model 
In rough terrain, the wheel can be modeled as a rigid wheel in deformable terrain (see Figure 8). A vertical load $W$ and a horizontal force $D P$ are applied to the wheel by the vehicle suspension. A torque $T$ is applied at the wheel rotation axis by an actuator. For this model, the wheel-terrain interaction forces can be given by [13]:

$$
\tau(\theta)=(c+\sigma(\theta) \tan \phi)\left(1-e^{-\frac{r}{k}\left[\theta_{1}-\theta-(1-i)\left(\sin \theta_{1}-\sin \theta\right)\right]}\right)
$$

where $c$ is the cohesion, is the internal friction angle, and $k$ is the shear deformation modulus. The normal stress at the wheel-terrain interface is:

$$
\sigma(z)=\left(k_{1}+k_{2} b\right)(z / b)^{n}
$$

where $b$ is the wheel width, $k_{1}$ and $k_{2}$ are constants, and $n$ is a sinkage coefficient.

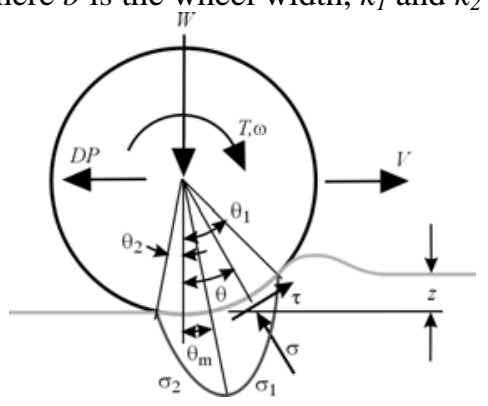

Fig. 8. Free-body diagram of rigid wheel on deformable terrain

It is assumed that the robot can sense terrain parameters as it travels. Methods have been developed for on-line terrain parameter estimation, using simple sensors [7]. Figure 9 shows a wheel-terrain interaction testbed to estimate terrain parameters, and a plot of experimental estimation of terrain cohesion and internal friction angle.
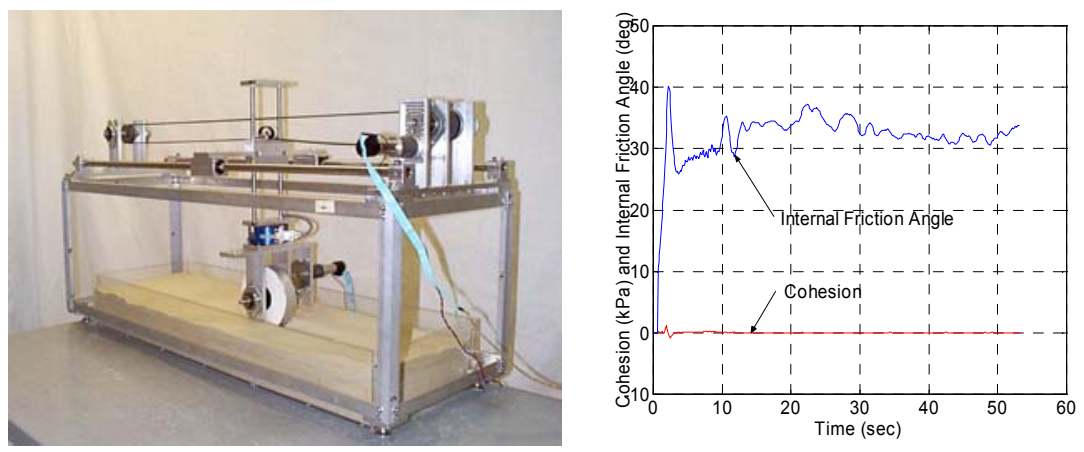

Fig. 9. Wheel-terrain interaction testbed and experimental terrain parameter estimation 


\section{Results}

Simulations and experiments were performed to examine the validity of the models to predict high-speed rough-terrain mobility. Simulations were compared to experimental results for the MOD 1 rover. A typical high-speed traverse of a 0.4 $\mathrm{m}$ wide ditch was examined, where the rover success is a function of its speed. The robot can successfully traverse the obstacle at low speed., see Figure 10. At high speed the robot can traverse the obstacle, by jumping over it, see Figure 12. However, at moderate speeds, the robot collides with the ditch edge, and is unable to complete the traverse, see Figure 11. The sensitivity of this obstacle to vehicle trajectory highlights the need for accurate model-based analysis.

Figure 10 shows photos of the experimental rover traversing the ditch at 1.4 $\mathrm{m} / \mathrm{s}$. In this series, the rover successfully traverses the obstacle. In contrast, Figure 11 shows a series of photos of the rover traversing the ditch at a moderate speed of $3.2 \mathrm{~m} / \mathrm{s}$. Here, the rover collides with the far edge of the ditch, and cannot extract itself. Figure 12 shows a series of photos of the experimental rover successfully traversing the ditch at approximately $7.4 \mathrm{~m} / \mathrm{s}$, by jumping over it.

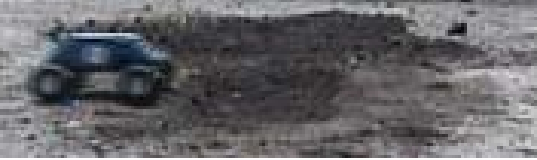

$\mathrm{t}=0 \mathrm{~s}$

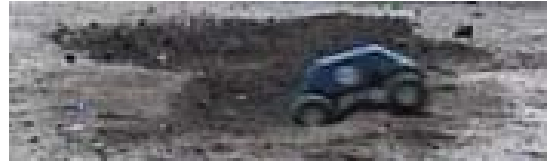

$\mathrm{t}=0.56 \mathrm{~s}$

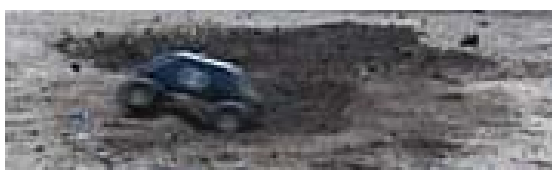

$\mathrm{t}=0.16 \mathrm{~s}$

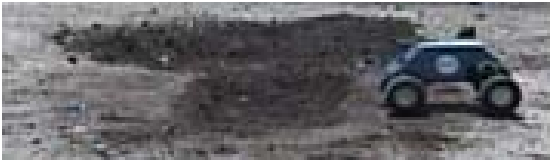

$\mathrm{t}=0.85 \mathrm{~s}$

Fig. 10. Photo series of low-speed ditch traversal $(1.4 \mathrm{~m} / \mathrm{s})$

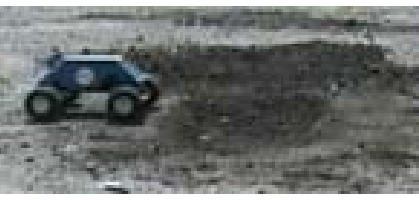

$\mathrm{t}=0 \mathrm{~s}$

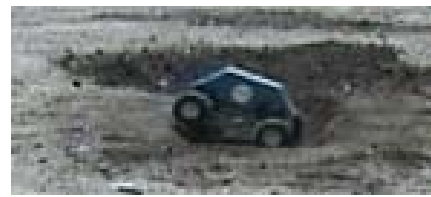

$0.16 \mathrm{~s}$

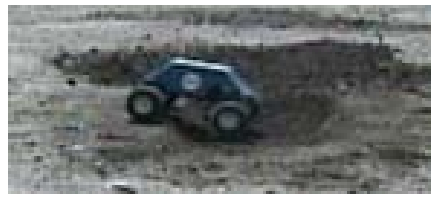

$\mathrm{t}=0.10 \mathrm{~s}$

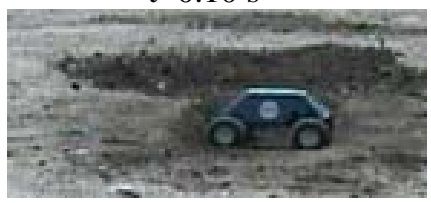

$\mathrm{t}=0.19 \mathrm{~s}$

Fig. 11. Photo series of moderate-speed ditch traversal $(3.2 \mathrm{~m} / \mathrm{s})$ 


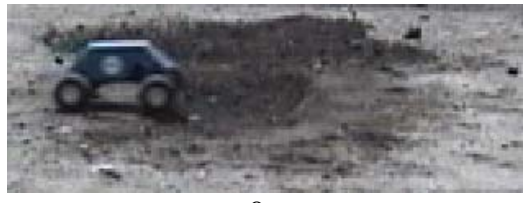

$\mathrm{t}=0 \mathrm{~s}$

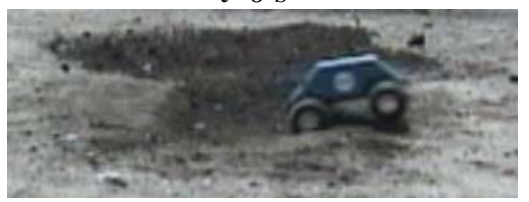

$\mathrm{t}=0.13 \mathrm{~s}$

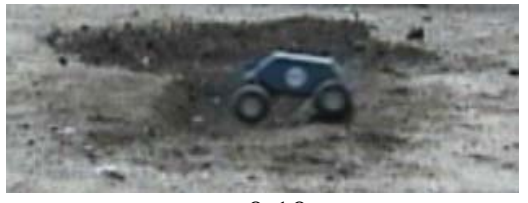

$\mathrm{t}=0.10 \mathrm{~s}$

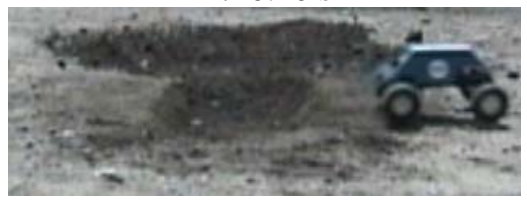

$\mathrm{t}=0.16 \mathrm{~s}$

Fig. 12. Photo series of high-speed ditch traversal $(7.4 \mathrm{~m} / \mathrm{s})$

Model-based analysis correctly predicted ditch traversability at low and high speeds (see Figure 13 for images from an ADAMS simulation). However, the model-based analysis was found to be highly sensitive to the terrain model. Using a simplified "force coefficient" model, the analysis gave poor prediction of the intermediate speed at which the ditch became nontraversable. This would suggest that modeling effort should focus on accurate representation of wheel-terrain interaction, an area which has been largely ignored in the mobile robotics literature. Also, as is obvious from Figures 10-13, vehicle ballistic behavior must be allowed in high-speed robot models, as well as wheel slip and deformation.
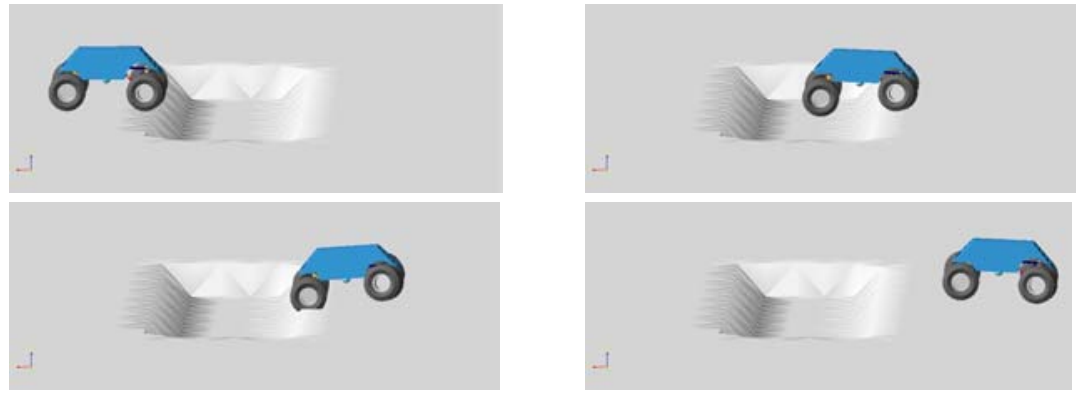

Fig. 13. Image series of high-speed ditch traversal simulation

\section{Conclusions and Lessons Learned}

In this paper, the concept of reactive behavior control has been presented, and an experimental high-speed mobile robot system has been described. Robot and terrain models have been presented. Simulation and experimental results for a fourwheel mobile robot performing a representative high-speed mobility task showed that complex models are required to accurately capture the motion dynamics of 
mobile robots in rough terrain. In particular, sensitivity to the wheel-terrain interaction model was observed. It was suggested that factors such as ballistic behavior, wheel slip, and wheel/terrain deformation must be considered in high-speed robot models.

\section{Acknowledgments}

The authors would like to acknowledge the support and assistance of the Defense Advanced Research Projects Agency (DARPA) and the U.S. Army TankAutomotive and Armaments Command (TACOM).

\section{References}

[1] Ben Amar, F. (1997) Steering Behavior and Control of Fast Wheeled Robots. In: Proceedings of the IEEE Conference on Intelligent Robots and Systems, IROS

[2] Cunningham, J., Roberts, J., Corke, P., and Durrant-Whyte, H. (1998) Automation of Underground LHD and Truck Haulage. In: Proceedings of Australian IMM Annual Conference, 241-246

[3] Frazzoli, E. (2001) Robust Hybrid Control for Autonomous Vehicle Motion Planning. Ph.D. Thesis, Massachusetts Institute of Technology

[4] Gerhart, G., Goetz, R., and Gorsich, D. (1999) Intelligent Mobility for Robotic Vehicles in the Army After Next. In: Proceedings of the SPIE Conference on Unmanned Ground Vehicle Technology, Vol. 3693

[5] Golombek, M. P. (1998) Mars Pathfinder Mission and Science Results. In: Proceedings of the 29th Lunar and Planetary Science Conference

[6] Gonthier, Y. and Papadopoulos, E. (1998) On the Development of a Real-time Simulator for an Electro-Hydraulic Forestry Machine. In: Proceedings of IEEE International Conference on Robotics and Automation

[7] Iagnemma, K., Shibly, H., and Dubowsky, S. (2002) On-Line Terrain Parameter Estimation for Planetary Rovers. In: Proceedings of the 2002 IEEE International Conference on Robotics and Automation

[8] Iagnemma, K., Genot, F., and Dubowsky, S. (1999) Rapid Physics-Based RoughTerrain Rover Planning with Sensor and Control Uncertainty. In: Proceedings of the 1999 IEEE International Conference on Robotics and Automation, pp. 2286 -2291

[9] Kelly, A., Stentz, A. (1998) Rough Terrain Autonomous Mobility - Part 2: An Active Vision, Predictive Control Approach. In: Autonomous Robots, Volume 5, pp. 163-198

[10] Osborn, J.F. (1989) Applications of Robotics in Hazardous Waste Management. In: Proceedings of the SME 1989 World Conference on Robotics Research

[11] Peng, H., and Tomizuka, M. (1993) Preview Control for Vehicle Lateral Guidance in Highway Automation. In: ASME Journal of Dynamic Systems, Measurement, and Control, Vol. 155

[12] Wong, J. (1993) Theory of Ground Vehicles. John Wiley and Sons

[13] Wong, J., and Reece, A (1967) Prediction of Rigid Wheels Performance Based on Analysis of Soil-Wheel Stresses, Part I. In: Journal of Terramechanics, Volume 4, Number 1, pp. 81-98 